\title{
Efeito do ibuprofeno administrado uma hora antes da inovulação de embriões bovinos
}

\author{
[Effect of ibuprofen administered one hour before the bovine embryo transfer] \\ H.J. Narváez ${ }^{1}$; R.S. Fontes ${ }^{2}$; R.L.D. Costa ${ }^{3}$, C.R. Quirino ${ }^{2}$, L.Z. Moreira ${ }^{4}$ \\ ${ }^{1}$ Aluno de pós-graduação - UENF - Campos dos Goytacazes, RJ \\ ${ }^{2}$ Centro de Ciências e Tecnologias Agropecuárias - UENF - Campos dos Goytacazes, RJ \\ ${ }^{3}$ APTA - Regional Extremo Oeste - Andradina, SP \\ ${ }^{4}$ Aluno de graduação - UENF - Campos dos Goytacazes, RJ
}

\begin{abstract}
RESUMO
Avaliou-se o efeito do ibuprofeno administrado uma hora antes da inovulação de embriões bovinos, com o objetivo de melhorar a taxa de prenhez. Após a avaliação da resposta ao protocolo de sincronização do estro, 76 fêmeas selecionadas como receptoras de embriões foram distribuídas em três grupos (G) experimentais: $\mathrm{G} 1 \quad(\mathrm{n}=25)$ receptoras usadas como controle, $\mathrm{G} 2(\mathrm{n}=30)$ receptoras que receberam ibuprofeno $5 \mathrm{mg} / \mathrm{kg}$, I.M, uma hora antes da inovulação dos embriões, e G3 (n=21) receptoras que receberam uma matriz polimérica de liberação controlada de ibuprofeno administrado por via subcutânea. As taxas de prenhez foram de 16\% (4/25), 43,3\% (13/30) e 14,2\% (3/21), para G1, G2 e G3, respectivamente. Observou-se diferença $(\mathrm{P}<0,024)$ na taxa de prenhez do $\mathrm{G} 2$ quando comparado ao $\mathrm{G} 1$ e ao G3. A administração do ibuprofeno por via intramuscular uma hora antes da inovulação dos embriões resultou em melhor taxa de prenhez em receptoras da raça Nelore.
\end{abstract}

Palavras-chave: bovino, transferência de embriões, anti-inflamatórios não esteroides, prostaglandinas, taxa de prenhez

\begin{abstract}
The effect of the administered ibuprofen was evaluated one hour before the embryo transfer of bovine embryos in order to improve pregnancy rates. After evaluating the response to protocol synchronization of estrus, 76 Females selected as the recipients of embryos were distributed into three experimental groups: $G 1(n=25)$ surrogate cows used as control, $G 2(n=30)$ surrogate cows that received $5 \mathrm{mg} / \mathrm{kg}$ ibuprofen, IM, one hour before the embryo transfer, and $G 3(n=20)$ surrogate cows that received an array polymeric release of controlled ibuprofen subcutaneously administered. The pregnancy rates were $16 \%$ (4/25), $43.3 \%$ (13/30), and 14.2\% (3/21) for G1, G2, and G3, respectively. There was statistical difference $(P<0.024)$ on pregnancy rate of $G 2$, in comparison with those of $G 1$ and $G 3$. The administration of ibuprofen intramuscularly one hour before the embryo transfer resulted in better pregnancy rate in Nellore surrogate cows.
\end{abstract}

Keywords: cattle, embryo transfer, non-steroidal anti-inflammatory, prostaglandin, pregnancy rates

\section{INTRODUÇÃO}

O ibuprofeno, potente anti-inflamatório não esteroide, derivado do ácido propiônico, é classificado como anti-inflamatório não seletivo. Possui a capacidade de inibir a atividade das duas isoformas da enzima ciclooxigenase (COX1 e COX-2) (Elli et al., 2001) e tem como função catalisar e transformar o ácido araquidônico em prostaglandinas (Radi e Khan, 2006).

$\mathrm{Na}$ reprodução de ruminantes, a prostaglandina $\mathrm{F}$ $2 \alpha$ (PGF $2 \alpha$ ) tem um reconhecido mecanismo que leva à regressão do corpo lúteo (CL) e, consequentemente, ao início de um novo ciclo estral (Odensvik et al., 1989). A administração

Recebido em 21 de setembro de 2009

Aceito em 10 de maio de 2010

E-mail: javiernarvaezvet@gmail.com 
de anti-inflamatórios, como substâncias inibidoras da síntese das prostaglandinas, vem sendo amplamente feita no momento da inovulação dos embriões em fêmeas receptoras das espécies bovina, ovina e caprina, com a finalidade de proteger a funcionalidade do CL e o desenvolvimento do embrião de uma possível liberação precoce de PGF2 $\alpha$ provocada pela manipulação do trato reprodutivo durante a inovulação dos embriões. Dessa forma, é possível obter melhores resultados nas taxas de prenhez em programas de transferência de embriões (TE) (Elli et al., 2001).

Alguns pesquisadores indicaram que a manipulação excessiva do trato reprodutivo pode acarretar trauma, seguido de processo inflamatório local, no qual é possível obter liberação de PGF2 $\alpha$ proveniente das células endometriais (Wann e Randel, 1990; Vélez et al., 1991; Scenna et al., 2005). Schrick et al. (2003) e Scenna et al. (2004) determinaram que a elevada concentração de PGF2 $\alpha$ no lume uterino, nos primeiros estádios da gestação, pode levar ao bloqueio do desenvolvimento embrionário ou retardo desse processo, reduzindo, assim, a habilidade do embrião de transição de estrutura pré-compacta a compacta.

Autores já evidenciaram o efeito do ibuprofeno e do flunixin meglumine (FM) na melhoria das taxas de prenhez, quando administrados em vacas e novilhas antes da inovulação dos embriões, ao demonstrarem a capacidade desses anti-inflamatórios em inibir a função da PGF2 $\alpha$ $\mathrm{e}$, assim, protegerem a funcionalidade do CL e a minimização dos efeitos deletérios de PGF2 $\alpha$ sobre a viabilidade do embrião (Elli et al., 2001; Purcell et al., 2005; Scenna et al., 2005).

Dessa forma, o trabalho teve como objetivo avaliar o efeito do ibuprofeno administrado uma hora antes da inovulação de embriões bovinos, visando melhorar as taxas de prenhez.

\section{MATERIAL E MÉTODOS}

O trabalho foi realizado nos meses de novembro de 2007 a fevereiro de 2008. De um total de 270 fêmeas solteiras disponíveis para pesquisa, foram selecionadas para o estudo 70 vacas Nelore Padrão e 30 novilhas Nelore Mocho, conforme a avaliação clínica geral, o exame ginecológico palpação via transretal dos órgãos genitais -, o peso e a qualificação do escore corporal na escala de 1 a 9 (Richard et al., 1986). Os animais selecionados foram mantidos em piquetes de capim Brachiaria decumbens, com suplementação mineral e água à vontade.

Para induzir à sincronização do estro, as fêmeas foram submetidas a tratamento hormonal em dia aleatório do ciclo estral (dia 0 ), com a colocação de um dispositivo intravaginal de $1,9 \mathrm{~g}$ de progesterona (CIDR - Pfizer, Brasil) mais uma aplicação intramuscular (IM) de $2 \mathrm{mg}$ de benzoato de estradiol (Cronibest - Biogenes, Brasil). No dia 8, o dispositivo intravaginal de progesterona foi removido, e administrou-se uma dose (IM) de $150 \mu \mathrm{g}$ de D-cloprostenol (Cronibem - PGF2 $\alpha$ - Biogenes). No dia 9, 24 horas após a aplicação de PGF2 $\alpha$, todos os animais receberam uma dose de $1 \mathrm{mg}$ de benzoato de estradiol IM. No sexto dia do ciclo estral, as receptoras foram submetidas a exame ultrassonográfico utilizando-se transdutor linear 5.0/7.5Mhz por via transretal (ScanVet200 - Pie Medical, Holanda), com a finalidade de avaliar a resposta das receptoras ao protocolo hormonal, com consequente formação de corpo lúteo (CL). Foram consideradas receptoras aptas para receber embrião somente as que apresentaram $\mathrm{CL} \geq 13 \mathrm{~mm}$ de diâmetro.

Os embriões inovulados pertenciam ao banco genético da APTA, Regional Extremo Oeste, Andradina, SP, que já haviam sido coletados e congelados em trabalhos anteriormente realizados, contendo os dados de qualidade e o estádio de desenvolvimento para cada estrutura. Os embriões encontravam-se congelados em etilenoglicol, crioprotetor que permite a transferência direta das estruturas. No dia da inovulação, dia 7 do ciclo estral, todas as receptoras foram submetidas à anestesia epidural baixa, $4 \mathrm{~mL}$ de cloridrato de lidocaína a $2 \%$, e à limpeza do reto e higienização da genitália externa com água e álcool 70\%. A inovulação dos embriões foi realizada por procedimento não cirúrgico no corno uterino ipsilateral ao ovário com corpo lúteo.

Foram avaliados a qualidade do embrião e o grau de manipulação do trato reprodutivo de acordo com a dificuldade de transposição do aplicador pela cérvice. Os critérios utilizados para qualificar a manipulação do trato reprodutivo foram semelhantes aos propostos por Scenna et 
al. (2005): grau 1, manipulação mínima do trato reprodutivo; grau 2, manipulação moderada; e grau 3, manipulação excessiva.

Uma hora antes da inovulação dos embriões, foram formados os três grupos (G) experimentais; as receptoras e os embriões foram aleatoriamente alocados dentro de cada grupo, com o objetivo de se evitar efeitos individuais nos resultados: G1 - controle, formado por animais que receberam $5 \mathrm{~mL}$ de solução salina fisiológica por via IM; G2 - (ou ibuprofeno-IM), animais que receberam $5 \mathrm{mg} / \mathrm{kg}$ IM de ibuprofeno (Henrifarma, Brasil) e G3 - (ou polímero-IB), animais que receberam uma matriz polimérica de ibuprofeno por via subcutânea (SC) na região lateral do pescoço (regio colli laterais).

Para a preparação da matriz polimérica contendo o ibuprofeno, realizou-se a seguinte metodologia: em um becker de $50 \mathrm{~mL}$, preparou-se uma solução aquosa de $80 \mathrm{~g}$ de pluronic F-127 (Sigma-Aldrich Chemical Co., EUA) e $80 \mathrm{~g}$ de PVA (Sigma-Aldrich) a uma concentração de $1,2 \%$ para cada um, sob agitação constante em agitador magnético a $400 \mathrm{x}$ g, por um minuto. Em outro becker de 50mL, dissolveu-se poli(lactídeo-co-glicolídeo) (PLGA - SigmaAldrich) em $5 \mathrm{~mL}$ de acetona a uma concentração de $2,9 \%$ massa/volume mais $15 \mathrm{~g}$ de ibuprofeno (Henrifarma). Utilizando-se um funil de vidro, verteram-se, lentamente, as duas soluções que foram submetidas à agitação mecânica a 400 x g, por um minuto (solvente + polímero + ibuprofeno). Em seguida, a emulsão foi mantida sob agitação severa por 12 horas para remoção total do solvente por evaporação. A emulsão restante foi centrifugada a $10.000 \mathrm{x} \mathrm{g}$, por 30 minutos. Ao final, a matriz polimérica, impregnada com ibuprofeno, foi lavada em água destilada por três vezes consecutivas e congelada a $-4^{\circ} \mathrm{C}$ até a aplicação (Azevedo, 2005). A matriz polimérica contendo o ibuprofeno foi administrada por via SC na região lateral do pescoço, com a finalidade de inibir qualquer possível liberação de PGF2 $\alpha$ nos primeiros estágios da gestação, desde a inovulação dos embriões até o mecanismo luteolítico, no $15^{\circ}$ dia do ciclo estral.

O diagnóstico da gestação foi realizado 35 a 40 dias após a inovulação dos embriões, por meio ultrassonográfico, utilizando um transdutor linear 5.0/7.5Mhz por via transretal.

A análise da taxa de aproveitamento foi realizada mediante a correção de Yates, para verificar as diferenças entre vacas e novilhas nas características: diâmetro do CL, peso e condição corporal. Foi realizada a análise de variância pelo procedimento GLM - SAS/1999 e aplicou-se o teste SNK $(\mathrm{P}<0,05)$ para comparar as médias. Nas análises de taxa de prenhez, qualidade do embrião e grau de manipulação do trato reprodutivo, utilizou-se o teste exato de Fisher (CHISQ/Fisher, SAS/1999)

\section{RESULTADOS E DISCUSSÃO}

Os resultados forneceram evidências de que a sincronização do estro e da ovulação em fêmeas da raça Nelore, utilizando-se um dispositivo intravaginal de progesterona associado ao benzoato de estradiol e PGF $2 \alpha$, proporcionou alta taxa da ovulação (90\% e $83,3 \%$, vacas e novilhas, respectivamente) determinada por ultrassonografia, verificado pela formação de CL. Das fêmeas utilizadas, $78,5 \%$ das vacas e $70,0 \%$ das novilhas foram classificadas como receptoras de embriões (Tab. 1).

Tabela 1. Taxa de aproveitamento (\%) e classificação do diâmetro do corpo lúteo (CL) conforme resposta ao protocolo de sincronização do estro

\begin{tabular}{ccccccc} 
Categoria & $\mathrm{N}$ & $\begin{array}{c}\text { Taxa de } \\
\text { aproveitamento }\end{array}$ & $\begin{array}{c}\text { CL1 } \\
(\geq 13 \mathrm{~mm})\end{array}$ & $\begin{array}{c}\text { CL2 } \\
(10-13 \mathrm{~mm})\end{array}$ & $\begin{array}{c}\text { CL3 } \\
(<10 \mathrm{~mm})\end{array}$ & N.O \\
\hline Vaca & 70 & $78,5 \mathrm{a}(55 / 70)$ & $80,0 \%(56 / 70)$ & $7,1 \%(5 / 70)$ & $2,8 \%(2 / 70)$ & $10,0 \%(7 / 70)$ \\
Novilha & 30 & $70,0 \mathrm{a}(21 / 30)$ & $80,0 \%(24 / 30)$ & $3,3 \%(1 / 30)$ & $0 \%$ & $16,6 \%(5 / 30)$ \\
\hline
\end{tabular}

$\mathrm{NO}=$ não ovularam. Não houve diferença entre vacas e novilhas $(\mathrm{P}>0,05)$.

Em CL1 ( $\geq 13 \mathrm{~mm})$, houve tendência de formação de CL de tamanho grande (Tab. 1). Os resultados são inconsistentes em relação aos relatados por Demczuk et al. (1998), no qual observaram maior incidência de CL de tamanho médio $(51,6 \%)$, em relação aos de tamanho grande e pequeno, $29,1 \%$ e $19,2 \%$, respectivamente. Coleman et al. (1987) e Spell et al. (2001) 
observaram maior formação de CL de tamanho grande em receptoras de embriões, resultados também observados neste estudo.

Das receptoras CL1, os valores quanto aos critérios de seleção são apresentados na Tab. 2 .
Foram observadas diferenças entre vacas e novilhas, devido ao estado reprodutivo.

Em relação à taxa de prenhez em G1, G2 e G3, os valores foram de 16\%, 43,3\% e 14,2\%, respectivamente. A diferença entre G2, em relação ao G1 e ao G3, foi significativa (Fig. 1).

Tabela 2. Valores (média \pm EPM) do diâmetro do corpo lúteo (CL), peso vivo e condição corporal das receptoras selecionadas após a sincronização do estro

\begin{tabular}{cccc}
\hline & & & \\
Categoria & Diâmetro do CL $(\mathrm{mm})$ & Peso $(\mathrm{kg})$ & Condição corporal \\
\hline Vaca & $17,41 \pm 0,57 \mathrm{a}$ & $500,69 \pm 5,93 \mathrm{a}$ & $7,47 \pm 0,48 \mathrm{a}$ \\
Novilha & $15,61 \pm 0,48 \mathrm{~b}$ & $356 \pm 7,01 \mathrm{~b}$ & $6,60 \pm 0,93 \mathrm{~b}$ \\
\hline
\end{tabular}

Médias seguidas por letras distintas, na coluna, diferem entre si pelo teste SNK $(\mathrm{P}<0,05)$.

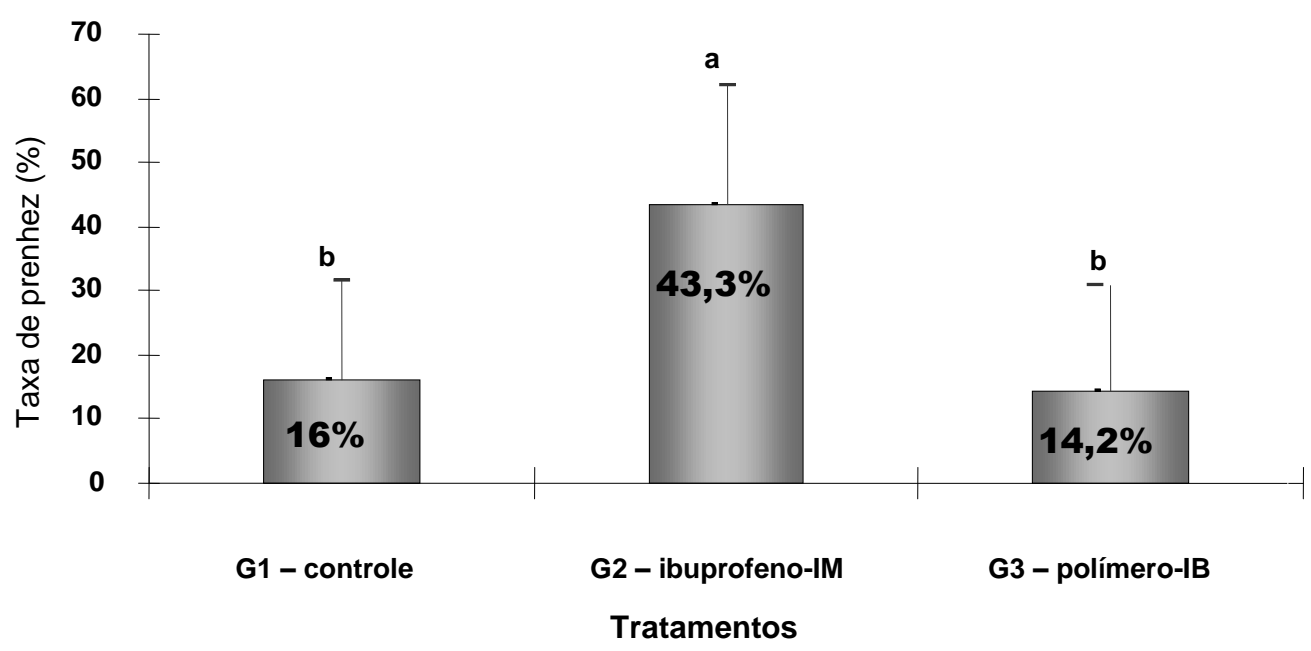

Figura 1. Taxa de prenhez de receptoras da raça Nelore conforme o grupo experimental.

Portanto, observou-se melhora de $27,3 \%$ na taxa de prenhez do grupo ibuprofeno-IM em relação ao grupo-controle, e de $29,1 \%$ em relação ao grupo polímero-IB, atribuída, provavelmente, à inibição da síntese de PGF2 $\alpha$ pela ação do ibuprofeno e à minimização dos efeitos deletérios de PGF2 $\alpha$ no desenvolvimento embrionário, o que proporcionou, assim, maior viabilidade ao embrião no mecanismo de implantação. É importante salientar que, neste trabalho, não foram quantificados os metabólitos de PGF $2 \alpha$ antes ou depois da inovulação dos embriões, o que poderia restringir a discussão dos dados. No entanto, com base em estudos que demonstraram metabólitos de PGF2 $\alpha$ antes e depois da inovulação dos embriões (Scenna et al., 2005) e a evidência das propriedades farmacológicas do ibuprofeno em inibir a síntese das prostaglandinas (Elli et al., 2001), é possível levar em consideração essas abordagens no entendimento dos resultados do presente estudo.

Acredita-se que a utilização do ibuprofeno com a finalidade de melhorar as taxas de prenhez em programas de TE, em bovinos, é feita com a aplicação do anti-inflamatório, uma única vez e, no mínimo, 30 minutos antes da inovulação dos embriões, considerando o tempo de absorção e a estabilização dos níveis sanguíneos requeridos.

Elli et al. (2001) avaliaram o efeito do ibuprofeno $5 \mathrm{mg} / \mathrm{kg}$ de peso vivo (IM) em novilhas da raça Holandesa, uma hora antes da inovulação dos embriões. Os embriões utilizados correspondiam a um programa de TE convencional e criopreservados, e a taxa de 
prenhez do grupo tratado com ibuprofeno foi diferente $(\mathrm{P}<0,05)$ em relação ao grupo-controle, $82 \%$ versus $56 \%$, respectivamente, demonstrando o efeito do ibuprofeno na melhora das taxas de prenhez em bovinos. Da mesma forma, Scenna et al. (2005) constataram o efeito $(\mathrm{P}<0,05)$ do flunixin meglumine $(\mathrm{FM})$ no acréscimo das taxas de prenhez em vacas e novilhas, utilizadas como receptoras de embriões frescos ou congelados.

Os resultados deste estudo diferem dos obtidos por McNaughtan (2004), o qual não encontrou efeito significativo na aplicação do FM antes da inovulação dos embriões em novilhas Hereford $\mathrm{x}$ Angus, embora o presente trabalho tenha utilizado o ibuprofeno como ferramenta farmacológica.

Quanto à taxa de prenhez do grupo que associou o ibuprofeno em uma matriz polimérica (polímero-IB), acredita-se que a desigualdade foi, possivelmente, em relação ao modelo de aplicação do ibuprofeno, que permite níveis plasmáticos do medicamento por longos períodos de tempo, porém inibe qualquer atividade das prostaglandinas, influenciando provavelmente importantes eventos nos primeiros estádios da gestação, como a vascularização endometrial, a eclosão do blastocisto, o mecanismo de implantação embrionária (Sayre e Lewis, 1993) e a funcionalidade do CL (Xiao et al., 1998).

Segundo Sayre e Lewis (1993) e Elli et al. (2001), estes importantes eventos da reprodução são dependentes de vários tipos de prostaglandinas e foram observados em várias espécies de mamíferos, incluindo a espécie humana e outros primatas. Portanto, diante do apresentado, sugere-se a importância de se manter a atividade das prostaglandinas nos primeiros estádios da gestação e de se evitar a administração de anti-inflamatórios por longos períodos de tempo após a inovulação dos embriões e até que ocorra o mecanismo de implantação embrionária.

Quanto ao efeito da qualidade do embrião sobre a taxa de prenhez (Tab. 3), observou-se diferença $(\mathrm{P}<0,05)$ entre $\mathrm{G} 2$ e $\mathrm{G} 1$ e $\mathrm{G} 3$, semelhante à tendência anterior em manter taxas de prenhez dos três grupos experimentais para os embriões classificados em grau II.

Tabela 3. Taxa de prenhez (média $\pm E P M)$ de acordo com a qualidade do embrião para cada grupo experimental

\begin{tabular}{lcccc}
\hline & & \multicolumn{3}{c}{ Taxa de prenhez $(\%)$} \\
\cline { 3 - 5 } \multicolumn{1}{c}{ Grupo } & $\begin{array}{c}\text { Taxa de } \\
\text { prenhez total }\end{array}$ & $\begin{array}{c}\text { Embrião grau I } \\
\text { n/n }\end{array}$ & $\begin{array}{c}\text { Embrião grau II } \\
\text { n/n }\end{array}$ & $\begin{array}{c}\text { Embrião grau III } \\
\text { n/n }\end{array}$ \\
\hline G1 - controle & $16 \%$ & $9,09 \pm 0,90 \mathrm{~b}(1 / 11)$ & $20 \pm 2,00 \mathrm{~b}(1 / 5)$ & $0 \mathrm{a}(0 / 2)$ \\
G2 - ibuprofeno-IM & $43,3 \%$ & $40 \pm 1,30 \mathrm{a}(6 / 15)$ & $62,50 \pm 1,82 \mathrm{a}(5 / 8)$ & $0 \mathrm{a}(0 / 3)$ \\
G3 - polímero-IB & $14,2 \%$ & $20 \pm 1,33 \mathrm{~b}(2 / 10)$ & $0 \mathrm{~b}(0 / 5)$ & $0 \mathrm{a}(0 / 3)$ \\
\hline
\end{tabular}

$\mathrm{IB}=$ ibuprofeno; $\mathrm{IM}=$ intramuscular.

Médias seguidas por letras distintas, na coluna, diferem entre si pelo teste exato de Fisher $(\mathrm{P}<0,05)$

Portanto, é possível considerar a utilização do ibuprofeno por via intramuscular para incrementar as taxas de prenhez de embriões grau I e II, principalmente, com maior aplicação para embriões grau II, pelo fato de apresentarem maior susceptibilidade aos efeitos deletérios de PGF2 $\alpha$ em relação aos embriões grau I. Estes resultados são semelhantes aos descritos por Scenna et al. (2005), que relataram melhor taxa de prenhez de embriões grau II do grupo tratado com FM em relação ao grupo-controle $(64,2 \%$ e $53,5 \%$, respectivamente). Em trabalhos anteriores, Elli et al. (2001), observaram aumento considerável nas taxas de prenhez para ambos os graus de qualidade (I e II) após a administração de ibuprofeno, semelhante aos resultados do presente estudo.

Com relação ao efeito do grau de manipulação do trato reprodutivo sobre as taxas de prenhez, observou-se diferença $(\mathrm{P}<0,05)$ entre $\mathrm{G} 1$ e $\mathrm{G} 2$ e G3 no grau de manipulação mínima e entre G2 e G1 e G3 no grau excessivo (Tab. 4). No grau 3 de manipulação do trato reprodutivo ocorreu no G2 uma notável diferença na taxa de prenhez, atribuída ao fato de este grupo ter recebido maior número de embriões de melhor qualidade, situação que refletiu nos resultados não esperados desta observação. 
Efeito do ibuprofeno administrado...

Tabela 4. Taxa de prenhez (médiaさEPM) de acordo com o grau de manipulação do trato reprodutivo para cada grupo experimental

\begin{tabular}{lcccc} 
Grupos de & & & Taxa de prenhez (\%) \\
\cline { 3 - 5 } tratamentos & $\mathrm{N}$ & 1-mínima (n) & 2-moderada (n) & 3-excessiva (n) \\
\hline G1 - controle & 25 & 0b (8) & $33,33 \pm 1,66 \mathrm{a}(9)$ & $12,50 \pm 1,25 \mathrm{~b}(8)$ \\
G2 - ibuprofeno-IM & 30 & $33,33 \pm 1,66 \mathrm{a}(9)$ & $30 \pm 1,52 \mathrm{a}(10)$ & $63,63 \pm 1,52 \mathrm{a}(11)$ \\
G3 - polímero-IB & 21 & $25 \pm 2,50 \mathrm{a}(4)$ & $28,57 \pm 1,84 \mathrm{a}(7)$ & $0 \mathrm{~b}(10)$ \\
\hline
\end{tabular}

IB = ibuprofeno; IM = intramuscular.

Médias seguidas por letras distintas, na coluna, diferem entre si pelo teste exato de Fisher $(\mathrm{P}<0,05)$.

A avaliação desse parâmetro em programas de TE poderia ser de grande importância, pois a maior manipulação do trato reprodutivo durante a inovulação dos embriões, por uma transposição mais trabalhosa do aplicador pela cérvice, pode ocasionar trauma no trato reprodutivo $\mathrm{e}$ desencadear processo inflamatório local, o qual levaria à liberação de PGF2 $\alpha$ com consequente comprometimento da gestação (Odensvik et al., 1993, Elli et al., 2001; Scenna et al., 2005). Segundo Scenna et al. (2005), a PGF2 $\alpha$, ao entrar em contato com o embrião, retarda ou bloqueia o desenvolvimento deste, diminuindo a habilidade de transição de estrutura pré-compacta a compacta (16 para 32 células) (Scenna et al., 2004) afetando de forma significativa a taxa de prenhez.

Conclui-se que a administração de ibuprofeno, por via intramuscular uma hora antes da inovulação dos embriões, melhora a taxa de prenhez em receptoras da raça Nelore. No entanto, a administração de ibuprofeno associado a uma matriz polimérica não aumenta o efeito sobre a taxa de prenhez.

\section{REFERÊNCIAS BIBLIOGRÁFICAS}

AZEVEDO, M.M.M. Sistemas poliméricos de liberação controlada utilizando micro $e$ nanopartículas encapsulando violaceína: caracterização, atividade biológica, consequências $e$ perspectivas. 2005. 177f. Tese (Doutorado) Universidade Estadual de Campinas, Campinas, SP.

COLEMAN, D.A.; DAILEY, R.A.; LEFFEL, R.E. et al. Estrous synchronization and establishment of pregnancy in bovine embryo transfer recipients. $J$. Dairy. Sci., v.70, p.858-866, 1987.

Arq. Bras. Med. Vet. Zootec., v.62, n.3, p.504-510, 2010
DEMCZUCK, E.; KOZICKI, L.E.; PONTELLI.; E.S. et al. Transferência de embrião em vacas da raça Simental na região noroeste do Paraná e sul do Mato Grosso do Sul. Braz. J. Vet. Res. Anim. Sci., v.35, p.174-177, 1998.

ELLI, M.; GAFFURI.; B.; FRIGERIO, A. et al. Effect of a single dose of ibuprofen lysinate before embryo transfer on pregnancy rates in cows. J. Reprod. Fertil., v.121, p.151-154, 2001.

McNAUGHTAN, J. The effect of prostaglandin inhibitor on pregnancy rates of heifer embryo transfer recipients. 2004. 38f. Dissertation (Master of Science) - Department of Plant \& Animal Sciences, Brigham Young University, Provo, EUA.

ODENSVIK, K.; CORT, N.; BASU, S. et al. Effect of flunixin meglumine on prostaglandin F2 $\alpha$ synthesis and metabolism in the pig. J. Vet. Pharm. Ther., v.12, p.307-311, 1989.

ODENSVIK, K.; DUCHENS, M.; GUSTAFSSON, H. Does mechanical manipulation of the reproductive organs cause a prostaglandin release in the heifer during embryo transfer? Act. Vet. Scand., v.34, p.219221, 1993.

PURCELL, S.H.; BEAL, W.E.; GRAY, K.R. Effect of a CIDR insert and flunixin meglumine, administered at the time of embryo transfer, on pregnancy rate and resynchronization of estrus in beef cattle. Theriogenology, v.64, p.867-878, 2005.

RADI, Z.A.; KHAN, N.K. Effects of cyclooxygenase inhibition on the gastrointestinal tract. Exp. Toxicol. Pathol., v.58, p.163-173, 2006.

RICHARDS, M.W.; SPITZER, J.C.; WARNER, M.B. Effect of varying levels postpartum nutrition body condition at calving on subsequent reproductive performance in beef cattle. J. Anim. Sci., v.62, p.301306,1986

SAYRE, B.L.; LEWIS, G.S. Arachidonic acid metabolism during early development of ovine embryos: a possible relationship to shedding of the zona pellucida. Prostaglandins, v.45, p.557-569, 1993. 
SCENNA，F.N.; EDWARDS, J.L.; ROHRBACH, N.R. et al. Detrimental effects of prostaglandin F2 $\alpha$ on preimplantation bovine embryos. Prost. Other Lipid Mediat., v.73, p.215-226, 2004

SCENNA, F.N.; HOCKETT, M.E.; TOWNS, T.M. et al. Influence of a prostaglandin synthesis inhibitor administered at embryo transfer on pregnancy rates of recipient cows. Prost. Other Lipid Mediat., v.78, p.3845, 2005.

SCHRICK, F.N.; SCENNA, F.N.; EDWARDS, J.L. et al. More evidence for a direct interaction between prostaglandin F2 $\alpha$ and development of bovine embryos. In: CETA AND AETA JOINT ANNUAL CONVENTION, 2003, Galgary, AB. Proceedings...Galgary, AB: CETA, 2003. p.43-52.

SPELL, A.R.; BEAL, W.E.; CORAH, L.R. et al. Evaluating recipient and embryo factors that affect pregnancy rates of embryo transfer in beef cattle. Theriogenology, v.56, p.287-297, 2001.
VELEZ, J.S.; RANDEL, R.D.; NEUENDORFF, D.A. Effect of uterine manipulation on postpartum fertility and plasma 13, 14-dihydro-15-keto prostaglandin F $2 \alpha$ in Brahman cows and first-calf heifers. Theriogenology, v.36, p.987-997, 1991.

WANN, R.A.; RANDEL, R.D. Effect of uterine manipulation 35 days after parturition on plasma concentrations of 13,14-dihydro-15-keto prostaglandin F2 $\alpha$ in multiparous and primiparous Brahman cows . J. Anim. Sci., v.68, p.1389-1394, 1990.

XIAO, C.; LIU, W.J.; SIROIS, J. et al. Regulation of cycloxigenase- 2 and prostaglandin $\mathrm{F}$ synthase gene expression by steroid hormones and interferon- $t$ in bovine epithelial cells. Endocrinology, v.139, p.22932299, 1998 